\title{
Treatment of Pneumocystis pneumonia with intermediate-dose and step-down to low-dose trimethoprim-sulfamethoxazole: lessons from an observational cohort study
}

\author{
Dina Creemers-Schild ${ }^{1}$ Frank P. Kroon ${ }^{1}$ Ed. J. Kuijper ${ }^{2}$ - Mark G. J. de Boer ${ }^{1}$
}

Received: 16 April 2015/Accepted: 7 August 2015/Published online: 15 October 2015

(C) The Author(s) 2015. This article is published with open access at Springerlink.com

\begin{abstract}
Background The recommended treatment of Pneumocystis jirovecii pneumonia (PCP) is high-dose trimethoprim-sulfamethoxazole (TMP-SMX) in an equivalent of TMP $15-20 \mathrm{mg} / \mathrm{kg} /$ day and SMX 75-100 mg/kg/day for 2 or 3 weeks. High rates of adverse events are reported with this dose, which raises the question if lower doses are possible.

Methods All adult patients diagnosed with PCP in various immune dysfunctions and treated with TMP-SMX between January 1, 2003 and July 1, 2013 in a tertiary university hospital were included. Per institutional protocol, patients initiated treatment on intermediate-dose TMP-SMX (TMP $10-15 \mathrm{mg} / \mathrm{kg} /$ day) and could be stepped down to low-dose TMP-SMX (TMP 4-6 mg/kg/day) during treatment. Clinical variables at presentation, relapse rate and mortality rates were compared between intermediate- and step-down treatment groups by uni- and multivariate analyses.

Results A total of 104 patients were included. Twentyfour patients $(23 \%)$ were switched to low-dose TMPSMX after a median of 4.5 days (IQR 2.8-7.0 days). One relapse $(4 \%)$ occurred in the step-down group versus none in the intermediate-dose group. The overall 30-day mortality was $13 \%$. There was 1 death in the step-down group
\end{abstract}

Mark G. J. de Boer

m.g.j.de_boer@lumc.nl

Dina Creemers-Schild

dinaschild@hotmail.com

1 Department of Infectious Diseases, Leiden University Medical Center, PO Box 9600, 2300 RC Leiden, The Netherlands

2 Department of Medical Microbiology, Leiden University Medical Center, Leiden, The Netherlands
(4\%) compared to 13 deaths $(16 \%)$ in the intermediatedose group.

Conclusions We observed high cure rates of PCP by treatment with intermediate-dose TMP-SMX. In addition, a step-down strategy to low-dose TMP-SMX during treatment in selected patients appears to be safe and does not compromise the outcome of treatment.

Keywords Pneumocystis jirovecii · Treatment · Low dose Cotrimoxazole $\cdot$ Trimethoprim-sulfamethoxazole . Antibiotic stewardship

\section{Introduction}

According to current guidelines and reviews, the recommended treatment of Pneumocystis jirovecii pneumonia (PCP) is high-dose trimethoprim-sulfamethoxazole (TMPSMX) in a dose of TMP $15-20 \mathrm{mg} / \mathrm{kg} /$ day and SMX $75-100 \mathrm{mg} / \mathrm{kg} /$ day for a duration of 2 or 3 weeks [1-3]. Most, if not all, of the evidence for this regimen is based on animal studies [4] and a small open label trial comparing high- versus low-dose TMP-SMX for the treatment of PCP in immunocompromised children [5]. In this clinical study performed almost 40 years ago, numerically more patients recovered on the high-dose regimen of TMP $20 \mathrm{mg} /$ $\mathrm{kg} /$ day-SMX $100 \mathrm{mg} / \mathrm{kg} / \mathrm{day}$ (11 out of 14) than when receiving TMP $4-7 \mathrm{mg} / \mathrm{kg} /$ day-SMX $20-35 \mathrm{mg} / \mathrm{kg} / \mathrm{day}$ (3 out of 6). In randomized trials comparing different therapeutic options for the treatment of HIV-associated PCP, high-dose TMP-SMX was always present as one of both treatment arms [6-10]. Irrespective of the response rates on high-dose TMP-SMX, high rates of adverse events and drug toxicity were observed. Rash, drug fever, neutropenia, renal insufficiency, electrolyte disorders and liver toxicity 
Table 1 Description of different TMP-SMX doses

\begin{tabular}{llll}
\hline & TMP $(\mathrm{mg} / \mathrm{kg} /$ day $)$ & SMX $(\mathrm{mg} / \mathrm{kg} /$ day $)$ & Standard dose used in the study \\
\hline High dose & $15-20$ & $75-100$ & Not used \\
Intermediate dose & $10-15$ & $50-75$ & $2400 \mathrm{mg}$ b.i.d. \\
Low dose & $4-6$ & $20-30$ & $960 \mathrm{mg}$ b.i.d. \\
\hline
\end{tabular}

were all encountered frequently. In the above-named trials, the severity of the side effects forced clinicians to switch to an alternative treatment (like pentamidine, dapsonetrimethoprim or clindamycin-primaquine) in over $30 \%$ of patients that were included in the high-dose TMP-SMX arms.

Limited retrospective data and one small randomized trial demonstrated that treatment of PCP with an intermediate dose of TMP-SMX (TMP $10 \mathrm{mg} / \mathrm{kg} /$ day and SMX $50 \mathrm{mg} / \mathrm{kg} /$ day) yielded comparable efficacy as the traditional high-dose regimen [11, 12]. Moreover, the intermediate dose was associated with lower rate of observed toxicity and treatment limiting adverse effects. The efficacy of treatment with low-dose TMP-SMX (TMP 4-6 mg/kg/day and SMX 20-30 mg/kg/day) has not been investigated in a clinical setting ever since the first introduction of TMP-SMX for the treatment of PCP by Hughes et al. in 1975. However, the safety and comparable efficacy of a step-down strategy to low-dose TMP-SMX after observation of adverse events was demonstrated in a small prospective study in 1990 [13].

Regarding the limited evidence from clinical studies as well as the high burden of drug toxicity and treatment limiting adverse events, rationale is provided for movement away from the generally recommended high-dose TMPSMX regimen. Since several years we routinely used in our institution an intermediate dose of TMP-SMX for all patients diagnosed with PCP. After starting this intermediate-dose regimen, it is an option for the attending clinician to switch to low-dose TMP-SMX based on observation of good clinical response or drug-related toxicity. In this study, we present the outcomes of this alternative treatment strategy. In addition, we assessed whether clinical characteristics at presentation may be indicative for later switching to low-dose TMP-SMX. In Table 1, an overview of the different dosing regimens referred to in this article is provided.

\section{Methods}

\section{Setting and study population}

The study was performed in the Leiden University Medical Center, a tertiary care and teaching hospital in the Netherlands with solid organ and haematological transplantation programs as well as providing care for HIVseropositive patients. The study was approved by the ethics committee of the Leiden University Medical Center. Written informed consent was not obtained, since patient information was anonymized and the methods of the study did not require informed consent under common law and ethics.

The databases of the Department of Medical Microbiology and the Pharmacy Department were cross-linked to identify all adult patients diagnosed with PCP and treated with TMP-SMX between January 1, 2003 and July 1, 2013. A confirmed diagnosis of PCP was defined as presence of respiratory symptoms, radiological evidence (interstitial pneumonitis on chest X-ray or computed tomography) and positive microbiological test, i.e. a positive polymerase chain reaction (PCR) and/or positive microscopy for $P$. jirovecii cysts by silver and Giemsa staining in bronchoalveolar lavage (BAL) fluid or induced sputum. Except for the implementation of a real-time-PCR method for detection of the dihydropteroate synthase (DHPS) coding region of $P$. jirovecii DNA in 2004 [14], the diagnostic and treatment protocols for PCP did not change during the study period. Confirmed cases were included in a retrospective cohort study. Patients that were $<18$ years old or who were treated with other regimens active against $P$. jirovecii were excluded from the analyses. Relapse of PCP was defined as a new diagnosis of PCP within 30 days after completion of at least a 2 -week course of treatment after which clinical cure was achieved. In case of relapse, only the first episode of PCP was included.

\section{Treatment conditions}

Initial, protocolled treatment for all patients diagnosed with PCP consisted of intermediate-dose TMP-SMX administered by oral or intravenous route twice daily. In Table 1, the different dosing regimens are specified. The standard duration of treatment was 2 weeks. Additional steroid therapy was started if the partial oxygen pressure was $<9.0 \mathrm{kPa}$. After commencing treatment with the intermediate dose, a step-down strategy to low-dose TMPSMX could be followed as per decision of the attending physician, usually after consultation with an infectious disease specialist or microbiologist. The decision would be conditional, based on observation of a favourable clinical 
response to initial therapy or in case of development of toxicity.

The intermediate-dose group consisted of patients who received at least 2 weeks of the intermediate dose of TMPSMX. Patients who were switched to low-dose TMP-SMX during treatment were included in the step-down group.

\section{Data collection and analyses}

For all included patients, clinical data about underlying disease, symptoms and laboratory values at presentation were extracted from the electronic patient files. Because the assay used to measure lactate dehydrogenase (LDH) levels was altered during the study period, we defined an elevated LDH as more than 1.5 times the upper limit of normal. Data concerning the diagnostic workup for PCP, the occurrence of ICU admission, length of hospitalization, 30-day relapse rate of PCP, 30- and 100-day all-cause mortality and recorded adverse events were documented.

Variables were compared between treatment groups. In addition, the 30-day mortality data were analysed in a caseby-case review. The cause of death was classified as either attributable to PCP (e.g. respiratory failure), contributable to PCP (e.g. secondary bacterial sepsis) or not related to PCP (e.g. gastrointestinal bleeding, myocardial infarction).

Statistical analyses were performed with the SPSS version 20.0 statistical package for Windows. Univariate risk factor analysis for binominal variables was performed using cross tables and Chi square statistical tests. Results are reported as risk ratios (RR) with $95 \%$ confidence intervals (CI95\%). For continuous variables, a Student's $t$ test or Mann-Whitney $U$ test was performed depending on the distribution of the respective variable. A $p$ value of $<0.05$ was considered to be significant. The observational study design leads to an inevitable confounding by indication. The effects of this bias and interpretation of results are discussed in detail in "Discussion".

\section{Results}

\section{Study cohort}

Out of 163 potential cases of PCP retrieved by query from the databases of the departments of medical microbiology and pharmacy, 132 patients with a confirmed diagnosis of PCP were identified. Ten patients were treated with a regimen other than TMP-SMX, eight patients received a dose of TMP-SMX that could not be classified per protocol in one of the two defined treatment groups and two patients were solely treated with low-dose TMP-SMX. One patient was a child. Seven patients prematurely terminated treatment before the end of 2 weeks. For five patients, this was

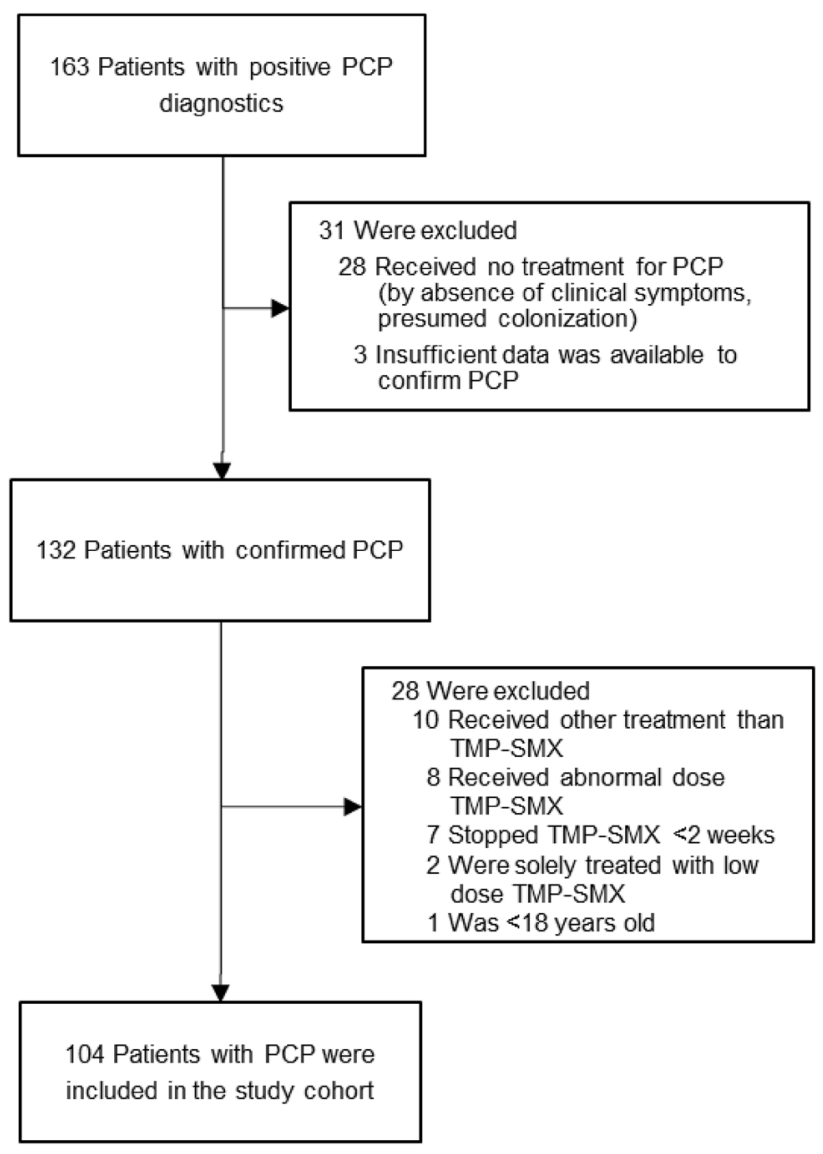

Fig. 1 The study cohort

due to toxicity and for two patients the reason was unknown. A total of 104 adult patients were included in the study cohort (Fig. 1).

Eighty patients $(77 \%)$ received the standard treatment of at least 2 weeks of intermediate-dose TMP-SMX. The majority of these patients ( 73 patients, $91 \%$ ) were treated for 2 weeks. Six patients were treated for 3 weeks and one patient was treated for 4 weeks. Twenty-four patients of the study cohort $(23 \%)$ were included in the step-down group. They were switched to low-dose TMP-SMX at a median of 4.5 days (IQR 2.8-7.0 days). In the whole study cohort, only one patient used TMP-SMX prophylaxis $(480 \mathrm{mg}$ o.d.) prior to diagnosis of PCP. This patient was recently diagnosed with HIV infection and had started taking prophylaxis only 15 days prior to the diagnosis of PCP.

Respiratory material was obtained from all patients as required by definition for a confirmed diagnosis of PCP. A BAL was performed in $99(95 \%)$ of the cases. In the remaining five patients, two patients in the step-down group and three patients in the intermediate-dose group, diagnostic tests were performed on sputum. Microscopy for $P$. jirovecii cysts was positive in 14 patients $(70 \%)$ in the step-down group and in 41 patients $(57 \%)$ in the intermediate-dose group. 
Table 2 Clinical characteristics at presentation per treatment schedule

\begin{tabular}{|c|c|c|c|c|}
\hline & $\begin{array}{l}\text { Step-down group } \\
(n=24)\end{array}$ & $\begin{array}{l}\text { Intermediate-dose group } \\
(n=80)\end{array}$ & $\begin{array}{l}\text { Risk ratio }^{\mathrm{a}}(95 \% \\
\text { CI) }\end{array}$ & $p$ value \\
\hline \multicolumn{5}{|l|}{ Clinical parameters at admission } \\
\hline Gender $\mathrm{M} / \mathrm{F}$ ratio & $16 / 8$ & $46 / 34$ & $1.36(0.64-2.87)$ & 0.42 \\
\hline Age (years) & $61(47-71)$ & $60(49-64)$ & & 0.43 \\
\hline Age $>50$ years & $17(71)$ & $59(74)$ & $0.89(0.46-1.93)$ & 0.78 \\
\hline Age $>65$ years & $9(38)$ & $21(44)$ & $1.55(0.77-3.14)$ & 0.23 \\
\hline Duration of respiratory symptoms at diagnosis & $7(3-19)$ & $10(3-14)$ & & 0.87 \\
\hline Temperature $\left({ }^{\circ} \mathrm{C}\right)$ & $37.8(37.3-38.8)$ & $37.7(37.0-38.3)$ & & 0.27 \\
\hline Respiratory rate (breaths per min) & $24(20-30)$ & $23(16-30)$ & & 0.33 \\
\hline \multicolumn{5}{|l|}{ Underlying condition } \\
\hline HIV infection & $5(21)$ & $12(15)$ & $1.35(0.58-3.11)$ & 0.50 \\
\hline Solid organ transplantation & $6(25)$ & $27(34)$ & $0.72(0.31-1.64)$ & 0.42 \\
\hline Haematology malignancy & $7(29)^{\mathrm{b}}$ & $25(31)$ & $0.93(0.43-2.01)$ & 0.85 \\
\hline Other & $7(29)$ & $16(20)$ & $1.44(0.68-3.07)$ & 0.34 \\
\hline \multicolumn{5}{|l|}{ Laboratory results } \\
\hline Leukocyte count $\left(\times 10^{9} / 1\right)$ & $7.2(3.6-10.9)$ & $7.2(4.8-11.3)$ & & 0.91 \\
\hline Leukocyte count elevated (>ULN) & $8(33)$ & $26(33)$ & $1.00(0.48-2.13)$ & 0.97 \\
\hline C-reactive protein $(\mathrm{mg} / \mathrm{l})$ & $74(36-153)$ & $92(48-194)$ & & 0.43 \\
\hline C-reactive protein $>100 \mathrm{mg} / \mathrm{l}$ & $10 / 21(48)$ & 28/61 (46) & $1.05(0.50-2.20)$ & 0.89 \\
\hline Creatinine $(\mu \mathrm{mol} / \mathrm{l})$ & $86(74-134)$ & $88(64-147)$ & & 0.52 \\
\hline Creatinine elevated ( $>1.5$ ULN) & $5(21)$ & $20(25)$ & $0.82(0.34-1.97)$ & 0.65 \\
\hline Lactate dehydrogenase elevated ( $>1.5$ ULN) & $7 / 23(30)$ & $42 / 78(54)$ & $0.46(0.21-1.03)$ & 0.048 \\
\hline $\mathrm{PaO}_{2}(\mathrm{kPa})$ & $7.9(6.5-9.2)$ & $7.9(6.9-8.8)$ & & 0.84 \\
\hline $\begin{array}{l}\text { Indication for steroid treatment }\left[\mathrm{PaO}_{2}\right. \\
\quad(\mathrm{kPa})<9.0]\end{array}$ & $11 / 16(69)$ & 48/61 (79) & $0.67(0.27-1.68)$ & 0.40 \\
\hline Start steroid schedule at diagnosis & $21 / 23(91)$ & $55 / 65(85)$ & $1.67(0.44-6.17)$ & 0.42 \\
\hline \multicolumn{5}{|l|}{ Outcome of diagnostic methods on BAL } \\
\hline PCR positive & $24(100)^{\mathrm{c}}$ & $76(100)^{\mathrm{d}}$ & - & - \\
\hline Microscopy positive & $14 / 20(70)$ & $41 / 72(57)$ & $1.49(0.66-3.72)$ & 0.29 \\
\hline$C_{\mathrm{t}}$ value & $33.7(30.4-34.6)$ & $34.1(30.7-37.1)$ & & 0.74 \\
\hline
\end{tabular}

Values represent median (IQR Q1-Q3) or no. (\%)

PCP Pneumocystis jirovecii pneumonia, CI confidence interval, HIV human immunodeficiency virus, $U L N$ upper limit of normal, BAL bronchoalveolar lavage, $P C R$ polymerase chain reaction, $I Q R$ interquartile range

\# $p$ value as calculated by Pearson Chi square test for bivariate variables and by Mann-Whitney $U$ test or independent samples Student's $t$ test (according to distribution) for continuous variables

${ }^{\text {a }}$ Risk ratios are for risk of switch to low-dose treatment

${ }^{\mathrm{b}}$ One patient in the step-down group with both non-Hodgkin lymphoma and a kidney transplantation

${ }^{c}$ In two cases, there was only a positive sputum PCR and no BAL performed

${ }^{\mathrm{d}}$ In three cases, there was only a positive sputum PCR and no BAL performed; in four cases PCR was not performed, but microscopy was positive

\section{Clinical characteristics at presentation}

The clinical characteristics at presentation with PCP are described in Table 2. The $\mathrm{LDH}$ serum level was more frequently elevated in the intermediate-dose group $(p=0.048)$. None of the other studied variables discriminated between the two treatment groups.

\section{Mortality and relapse}

The 30-day all-cause mortality was $13 \%$ (14 patients). There was one death in the step-down group (4\%) versus 13 deaths $(16 \%)$ in the intermediate-dose group $(p=0.13$, Table 3 ). The 14 patients with 30-day mortality are shown in Table 4. The patients died at a median of 14 days (IQR 
Table 3 ICU admission, hospital stay, toxicity and outcome of patients with PCP

\begin{tabular}{|c|c|c|c|c|}
\hline & $\begin{array}{l}\text { Step-down group } \\
(n=24)\end{array}$ & $\begin{array}{l}\text { Intermediate-dose group } \\
(n=80)\end{array}$ & $\begin{array}{l}\text { Risk ratio }{ }^{\mathrm{a}}(95 \% \\
\text { CI) }\end{array}$ & $p$ value $^{\#}$ \\
\hline \multicolumn{5}{|l|}{ ICU admission and hospital stay } \\
\hline ICU admission & $4(17)$ & $26(33)$ & $0.49(0.18-1.32)$ & 0.13 \\
\hline Length of hospitalization (days) & $15(9-24)$ & $15(8-33)$ & & 0.91 \\
\hline \multicolumn{5}{|l|}{ Toxicity } \\
\hline $\begin{array}{l}\text { (Pre-switch) in file reported TMP-SMX } \\
\text { toxicity }\end{array}$ & $5(21)$ & $4(5)$ & $2.78(1.37-5.65)$ & 0.02 \\
\hline \multicolumn{5}{|l|}{ Outcome } \\
\hline 30 -day relapse rate & $1(4)$ & 0 & - & 0.06 \\
\hline 30-day mortality & $1(4)$ & $13(16)$ & $0.28(0.04-1.91)$ & 0.13 \\
\hline 100-day mortality & $2(8)$ & $20(25)$ & $0.34(0.09-1.33)$ & 0.08 \\
\hline
\end{tabular}

Values are median (IQR Q1-Q3) or no. (\%)

PCP Pneumocystis jirovecii pneumonia, CI confidence interval, ICU intensive care unit, IQR interquartile range

\# $p$ value as calculated by Pearson Chi square test for bivariate variables and by Mann-Whitney $U$ test or independent samples Student's $t$ test (according to distribution) for continuous variables

${ }^{a}$ Risk ratios are for risk of switch to low-dose treatment

9-18) after diagnosis of PCP. In two patients (14\%), the mortality was not related to PCP (1 patient with multiorgan failure and upper gastrointestinal bleeding and 1 patient with hypovolemic shock due to abdominal bleeding). In three patients ( $21 \%)$, PCP contributed to mortality and in eight patients (57\%) death was attributable to PCP. One patient died of unknown cause. In the only patient who died in the step-down group, death was attributable to PCP.

The 100-day mortality was $8 \%$ (2 patients) and $25 \%$ (20 patients) for the step-down and intermediate-dose groups, respectively $(p=0.08)$.

There was one relapse in the low-dose group occurring 19 days after the initial episode. In this patient, the differential diagnosis was immune reconstitution inflammatory syndrome (IRIS) 10 days after starting combination antiretroviral therapy (cART) for a newly diagnosed HIV and PCP. In the second episode he recovered with steroid treatment only, although $P$. jirovecii was again detected in a fluid specimen from a second BAL.

Of note, the median length of hospitalization was 14.5 days in the step-down group versus 15 days in the intermediate-dose group $(p=0.91)$. ICU admission tended to be less frequent in the step-down group (17 vs. $33 \%$, $p=0.13$ ). In a multivariate analysis adjusted for age and co-morbidity, CRP levels and the partial oxygen pressure at presentation were variables independently associated with later admission to the ICU (data not shown).

\section{Toxicity}

Toxicity was more frequently reported in the step-down group, five patients in the step-down group (21\%) versus four patients in the intermediate-dose group (5\%). The reported toxicity included rash (5 patients), hepatotoxicity (2 patients), hyperkalaemia (2 patients) and renal insufficiency (1 patient). Toxicity was the reason to switch to low-dose TMP-SMX for the five patients in the step-down group after a median of 7 days of treatment. These five patients (4 with rash, 1 with elevated liver enzymes) continued their low-dose treatment with satisfactory results.

\section{Discussion}

\section{TMP-SMX doses and treatment outcome of PCP}

The results suggest that treatment of PCP with an intermediate dose of TMP-SMX has comparable efficacy as the recommended high-dose regimen. In addition, a step-down to low-dose TMP-SMX is likely an option for patients with a favourable clinical course and/or patients who already developed TMP-SMX-related toxicity. There was no increase in treatment failure or relapse in the step-down group.

Our experience with the intermediate dose of TMPSMX complies with two previous studies in which a similar intermediate-dose regimen was used [11, 12]. Thomas et al. conducted a retrospective review of $73 \mathrm{HIV}$-infected patients with PCP treated with TMP $10 \mathrm{mg} / \mathrm{kg} / \mathrm{day}$ and SMX $50 \mathrm{mg} / \mathrm{kg} /$ day. The relapse rate was $7 \%$. A low mortality rate of $7 \%$ was reported, though ICU admission was only indicated in $12 \%$ of cases. Bowden et al. conducted a randomized trial of $30 \mathrm{HIV}$-infected patients who received either TMP $20 \mathrm{mg} / \mathrm{kg} /$ day-SMX $100 \mathrm{mg} / \mathrm{kg} /$ day 


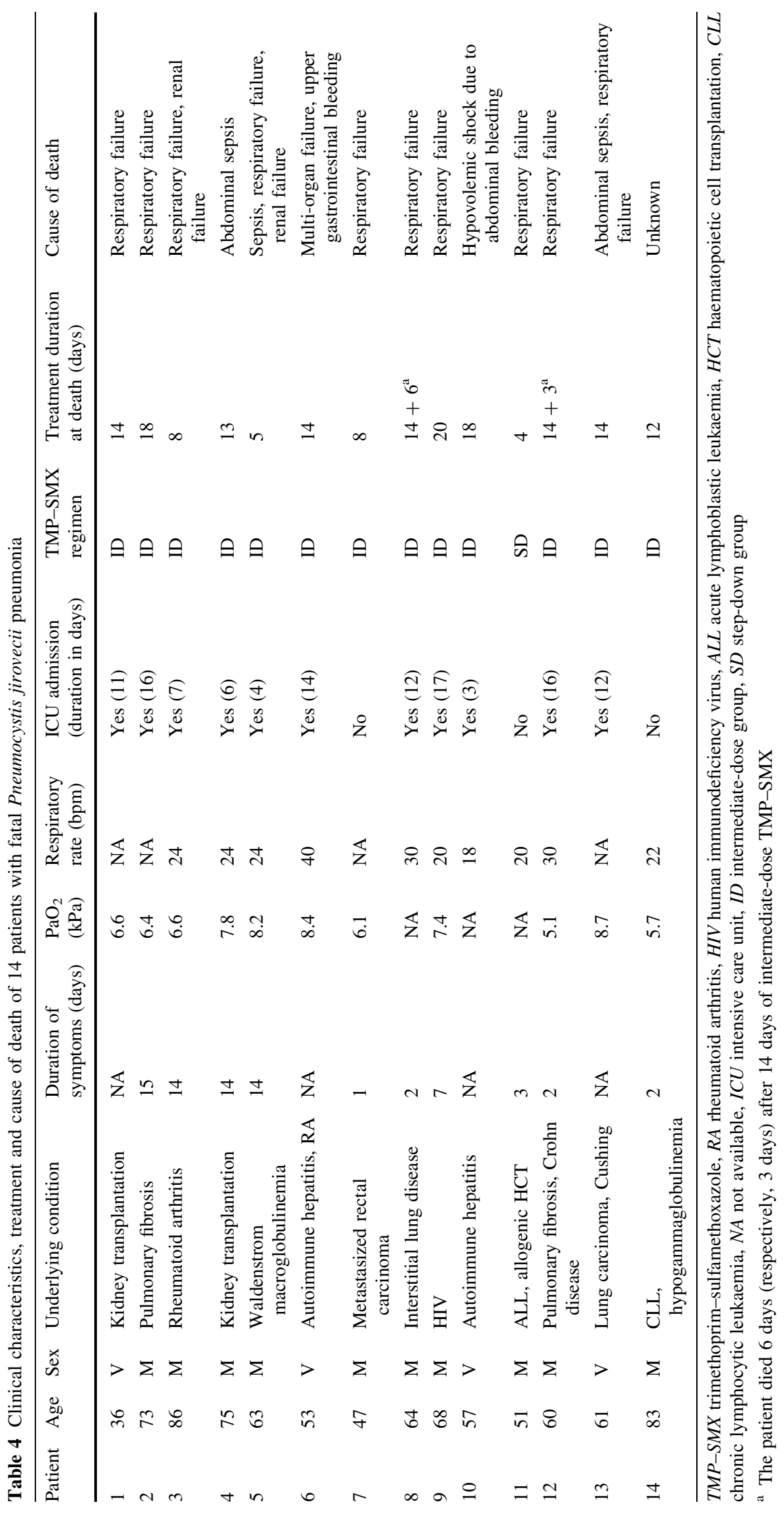


or TMP $10 \mathrm{mg} / \mathrm{kg} /$ day-SMX $50 \mathrm{mg} / \mathrm{kg} / \mathrm{day}$, with no differences in treatment failures. Mortality was not reported, but only patients with mild PCP were included. To the best of our knowledge, there is only one previous study that investigated a step-down regimen of TMP-SMX for the treatment of PCP [13]. Eeftinck Schattenkerk et al. retrospectively reviewed the case records of 50 patients with AIDS and PCP in the era prior to both cART and additional steroid therapy in the treatment of PCP. When drug intolerance developed, patients were switched to maintenance dose TMP-SMX (TMP 160 mg and SMX 800 mg o.d.). In this study, 20 patients were switched to maintenance-level TMP-SMX after an average of 9.6 days. There was no relapse in this group and overall survival was comparable with the group that received 2 weeks of high-dose TMPSMX.

In contrast to this approach, the current protocol allowed patients with improving condition to switch also if no adverse events due to TMP-SMX were observed. Nevertheless, more adverse events were reported in the step-down group than in the intermediate-dose group, suggesting that the appearance of toxicity was an important incentive for physicians to pursue a switch to low-dose TMP-SMX. After the appearance of TMP-SMX toxicity, five patients were able to complete their treatment with low-dose TMPSMX, thereby avoiding the use of alternative regimens like pentamidine or clindamycin plus primaquine.

Our study cohort contains many severe cases of PCP, i.e. ICU admission was required in $29 \%$ of cases. The overall 30 -day mortality rate of $13 \%$ in our study is comparable to or even lower than the mortality rates of 12-18\%, reported from earlier studies. In these studies, a high dose of TMPSMX was used for HIV-related PCP, with concomitant additional steroid treatment if indicated [7, 15-18]. Mortality rates for PCP in immunocompromised HIV-negative patients have been found to be even higher (35-50 \%) [1921].

\section{Clinical parameters and step-down to low-dose TMP-SMX}

The current study did not identify the clinical characteristics at the time of diagnosis that were predictive of a successful switch to low-dose TMP-SMX. Lower levels of LDH were present in the step-down group, but this parameter was not sufficiently discriminative. Notably, clinical variables known to be predictive for an unfavourable outcome, e.g. age, partial oxygen pressure and high C-reactive protein (CRP) [22-24], were not different between the two treatment groups. In a separate analysis (data not shown), the correlation between mortality and high CRP as well as low partial oxygen pressure at presentation was confirmed in our cohort. Several factors may explain the fact that the clinical characteristics-at presentation-usually associated with a favourable outcome were not found to be associated with a switch to low-dose treatment. First, a subcategory of patients that continued treatment on intermediate-dose TMP-SMX might have done well also after switch to low-dose therapy. The opportunity to switch may simply have been missed by the attending physicians. Secondly, the decision to switch to low-dose therapy was made during the course of treatment and not at presentation. Reassessment of clinical variables at the time of consideration of decreasing the dose of TMP-SMX could more accurately disclose which clinical variables predict a successful step-down strategy.

\section{Pharmacology of TMP-SMX and treatment of PCP}

Because of the difficulties of propagating $P$. jirovecii for a prolonged time in vitro, minimal inhibitory concentrations (MICs) and clinical breakpoints have not been obtained for TMP-SMX with regard to treatment of PCP [25-27]. Data from animal studies on this subject are available but concern different Pneumocystis species, e.g. P. carinii [4]. The results of these investigations may not be easily extrapolated to PCP in humans. TMP-SMX has excellent bioavailability, nearly $100 \%$ after oral administration [28]. For trimethoprim, serum levels of $2 \mu \mathrm{g} / \mathrm{ml}$ are reached after ingestion of $960 \mathrm{mg}$ of TMP-SMX, and remain stable for approximately $6 \mathrm{~h}$, after which the concentration steadily falls [29]. The TMP to SMX concentration ratio in cotrimoxazole is 1:5, but in serum it reaches a concentration ratio of 1:20 and both compounds are bound to serum proteins for approximately two-thirds of their total concentration [30]. As a consequence, a dose of $960 \mathrm{mg}$ cotrimoxazole b.i.d. would approximately result in effective (i.e. unbound) concentrations of 0.67 and $13.3 \mu \mathrm{g} / \mathrm{ml}$ of TMP and SMX, respectively, for 12 per $24 \mathrm{~h}$. From in vitro studies, the estimates of the $\mathrm{IC}_{90}$ of SMX for P. carinii (i.e. rat Pneumocystis) were found to be $8 \mu \mathrm{g} / \mathrm{ml}$ (trophozoites) and $16 \mu \mathrm{g} / \mathrm{ml}$ (cysts) when combined with $0.5 \mu \mathrm{g} / \mathrm{ml}$ TMP [31]. Although results of such experiments have not been published for $P$. jirovecii, this suggests that effective killing of $P$. jirovecii for over $50 \%$ of the time treated with cotrimoxazole $960 \mathrm{mg}$ b.i.d. is expected. However, further in vitro study of $P$. jirovecii susceptibility is needed to provide a stronger pharmacological basis for the rationale of treatment with low-dose TMPSMX. Due to good tissue penetration, concentrations of TMP and SMX in organs have frequently been found to be higher than serum concentrations [32, 33].

\section{Study limitations and strengths}

Because of its non-randomized design, equal effectiveness or non-inferiority of the executed strategy cannot be 
claimed. The obvious confounding by indication also explains the higher mortality in the intermediate-dose group, and patients with a favourable clinical course would be more prevalent in the step-down group. In addition, toxicity was an important reason for the attending physician to switch to low-dose TMP-SMX, accounting for the high frequency of reported toxicity in the step-down group.

The study included a substantial number of patients and the clinical practice setting is representative of standard diagnostic workup and care for patients with PCP. Different groups of immunocompromised patients are equally represented in both groups. This emphasizes that the stepdown approach was followed regardless of the underlying immunodeficiency of the patient. Therefore, differences between PCP in HIV and non-HIV infected patients probably do not impair the interpretation of the results [34, 35].

\section{Conclusions}

Despite the observational design of the study, the results strongly suggest that treatment of PCP with intermediatedose TMP-SMX has a similar efficacy compared to treatment with the recommended high-dose regimen. A randomized controlled trial would be necessary to provide clear evidence that both regimens are equally effective. Since the superiority of the high-dose regimen over an intermediate-dose or even over a low-dose regimen was never established by modern epidemiological standards in any clinical trial, the standard use of an intermediate-dose TMP-SMX as PCP therapy is-at the very least-open for discussion. The issue of lower frequency of severe toxic side effects, i.e. neutropenia, drug-induced hepatitis and rash, observed in patients who use an intermediate dose herein plays an important role.

In addition, a consecutive step-down strategy to lowdose TMP-SMX appears to be successful in selected patients, regardless of underlying disease and clinical characteristics at presentation. In case of development of toxicity during treatment with intermediate-dose TMPSMX, our results confirm that most of these patients are able to complete treatment with low-dose TMP-SMX.

Further studies should focus on optimizing the balance between efficacy and toxicity of TMP-SMX by patienttailored treatment of PCP.

\section{Compliance with ethical standards}

Conflict of interest The authors declare that they have no conflict of interest.

Open Access This article is distributed under the terms of the Creative Commons Attribution 4.0 International License (http:// creativecommons.org/licenses/by/4.0/), which permits unrestricted use, distribution, and reproduction in any medium, provided you give appropriate credit to the original author(s) and the source, provide a link to the Creative Commons license, and indicate if changes were made.

\section{References}

1. Panel on Opportunistic Infections in HIV-Infected Adults and Adolescents. Guidelines for the prevention and treatment of opportunistic infections in HIV-infected adults and adolescents: recommendations from the Centers for Disease Control and Prevention, the National Institutes of Health, and the HIV Medicine Association of the Infectious Diseases Society of America. http://aidsinfo.nih.gov/contentfiles/lvguidelines/adult_oi.pdf. Accessed 18 June 2014.

2. Walzer PD, Smulian AG. Pneumocystis species. In: Mandell GL, Bennett JE, Dolin R, editors. Mandell, Douglas, and Bennett's principles and practice of infectious diseases, 7th ed. Philadelphia; 2010. p. 3377-90.

3. Gilroy SA, Bennett NJ. Pneumocystis pneumonia. Semin Respir Crit Care Med. 2011;32:775-82.

4. Hughes WT, McNabb PC, Makres TD, Feldman S. Efficacy of trimethoprim and sulfamethoxazole in the prevention and treatment of Pneumocystis carinii pneumonitis. Antimicrob Agents Chemother. 1974;5:289-93.

5. Hughes WT, Feldman S, Sanyal SK. Treatment of Pneumocystis carinii pneumonitis with trimethoprim-sulfamethoxazole. Can Med Assoc J. 1975;112:47S-50S.

6. Wharton JM, Coleman DL, Wofsy CB, Luce JM, Blumenfeld W, Hadley WK, Ingram-Drake L, Volberding PA, Hopewell PC. Trimethoprim-sulfamethoxazole or pentamidine for Pneumocystis carinii pneumonia in the acquired immunodeficiency syndrome. A prospective randomized trial. Ann Intern Med. 1986;105:37-44.

7. Sattler FR, Frame P, Davis R, Nichols L, Shelton B, Akil B, Baughman R, Hughlett C, Weiss W, Boylen CT, van der Horst C, Black J, Powderly W, Steigbigel RT, Leedom JM, Masur H, Feinberg J. Trimetrexate with leucovorin versus trimethoprimsulfamethoxazole for moderate to severe episodes of Pneumocystis carinii pneumonia in patients with AIDS: a prospective, controlled multicenter investigation of the AIDS Clinical Trials Group Protocol 029/031. J Infect Dis. 1994;170:165-72.

8. Klein NC, Duncanson FP, Lenox TH, Forszpaniak C, Sherer CB, Quentzel H, Nunez M, Suarez M, Kawwaff O, Pitta-Alvarez A, Freeman K, Wormser GP. Trimethoprim-sulfamethoxazole versus pentamidine for Pneumocystis carinii pneumonia in AIDS patients: results of a large prospective randomized treatment trial. AIDS. 1992;6:301-5.

9. Safrin S, Finkelstein DM, Feinberg J, Frame P, Simpson G, Wu A, Cheung T, Soeiro R, Hojczyk P, Black JR. Comparison of three regimens for treatment of mild to moderate Pneumocystis carinii pneumonia in patients with AIDS. A double-blind, randomized, trial of oral trimethoprim-sulfamethoxazole, dapsonetrimethoprim, and clindamycin-primaquine. ACTG 108 Study Group. Ann Intern Med. 1996;124:792-802.

10. Hughes W, Leoung G, Kramer F, Bozzette SA, Safrin S, Frame P, Clumeck N, Masur H, Lancaster D, Chan C, Lavelle J, Rosenstock J, Falloon J, Feinberg J, Lafon S, Rogers M, Sattler F. Comparison of atovaquone (566C80) with trimethoprim-sulfamethoxazole to treat Pneumocystis carinii pneumonia in patients with AIDS. N Engl J Med. 1993;328:1521-7.

11. Thomas M, Rupali P, Woodhouse A, Ellis-Pegler R. Good outcome with trimethoprim $10 \mathrm{mg} / \mathrm{kg} / \mathrm{day}$-sulfamethoxazole $50 \mathrm{mg} /$ 
kg/day for Pneumocystis jirovecii pneumoniae in HIV infected patients. Scand J Infect Dis. 2009;41:862-8.

12. Bowden FJ, Stewart K, Mashford L, Lucas CR. A randomised, double blind trial of low dose versus high dose cotrimoxazole in the treatment of aids-related Pneumocystis carinii pneumonia. Aust N Z J Med. 1991;21:593.

13. Eeftinck Schattenkerk JK, Lange JM, van Steenwijk RP, Danner SA. Can the course of high dose cotrimoxazole for Pneumocystis carinii pneumonia in AIDS be shorter? A possible solution to the problem of cotrimoxazole toxicity. $J$ Intern Med. 1990;227:359-62.

14. Linssen CF, Jacobs JA, Beckers P, Templeton KE, Bakkers J, Kuijper EJ, Melchers WJ, Drent M, Vink C. Inter-laboratory comparison of three different real-time PCR assays for the detection of Pneumocystis jirovecii in bronchoalveolar lavage fluid samples. J Med Microbiol. 2006;55:1229-35.

15. Benfield TL, Helweg-Larsen J, Bang D, Junge J, Lundgren JD. Prognostic markers of short-term mortality in AIDS-associated Pneumocystis carinii pneumonia. Chest. 2001;119:844-51.

16. Dworkin MS, Hanson DL, Navin TR. Survival of patients with AIDS, after diagnosis of Pneumocystis carinii pneumonia, in the United States. J Infect Dis. 2001;183:1409-12.

17. Pulvirenti J, Herrera P, Venkataraman P, Ahmed N. Pneumocystis carinii pneumonia in HIV-infected patients in the HAART era. AIDS Patient Care STDS. 2003;17:261-5.

18. Rahdi S, Alexander T, Ukwu M, Saleh S, Morris A. Outcome of HIV-associated Pneumocystis pneumonia in hospitalized patients from 2000 through 2003. BMC Infect Dis. 2008;8:118-9.

19. Arend SM, Kroon FP, van't Wout JW. Pneumocystis carinii pneumonia in patients without AIDS, 1980 through 1993. An analysis of 78 cases. Arch Intern Med. 1995;1995:2436-41.

20. Roblot F, Godet C, Le Moal G, Garo B, Faouzi Souala M, Dary M, De Gentile L, Gandji JA, Guimard Y, Lacroix C, Roblot P, Becq-Giraudon B. Analysis of underlying diseases and prognosis factors associated with Pneumocystis carinii pneumonia in immunocompromised HIV-negative patients. Eur J Clin Microbiol Infect Dis. 2002;21:523-31.

21. Sepkowitz KA, Brown AE, Telzak EE, Gottlieb S, Armstrong D. Pneumocystis carinii pneumonia among patients without AIDS at a cancer hospital. JAMA. 1992;267:832-7.

22. Armstrong-James D, Copas AJ, Walzer PD, Edwards SG, Miller RF. A prognostic scoring tool for identification of patients at high and low risk of death from HIV-associated Pneumocystis jirovecii pneumonia. Int J STD AIDS. 2011;22:628-34.
23. Sage EK, Noursadeghi M, Evans HE, Parker SJ, Copas AJ, Edwards SG, Miller RF. Prognostic value of C-reactive protein in HIV-infected patients with Pneumocystis jirovecii pneumonia. Int J STD AIDS. 2010;21:288-92.

24. Fei MW, Kim EJ, Sant CA, Jarlsberg LG, Davis JL, Swartzman A, Huang L. Predicting mortality from HIV-associated Pneumocystis pneumonia at illness presentation: an observational cohort study. Thorax. 2009;64:1070-6.

25. Sloand E, Laughon B, Armstrong M, Bartlett MS, Blumenfeld W, Cushion M, Kalica A, Kovacs JA, Martin W, Pitt E, Pesanti EL, Richards F, Rose R, Walzer P. The challenge of Pneumocystis carinii culture. J Eukaryot Microbiol. 1993;40:188-95.

26. Thomas CF Jr, Limper AH. Current insights into the biology and pathogenesis of Pneumocystis pneumonia. Nat Rev Microbiol. 2007;5:298-308.

27. Merali S, Frevert U, Williams JH, Chin K, Bryan R, Clarkson AB Jr. Continuous axenic cultivation of Pneumocystis carinii. Proc Natl Acad Sci USA. 1999;96:2402-7.

28. Chin TW, Vandenbroucke A, Fong IW. Pharmacokinetics of trimethoprim-sulfamethoxazole in critically ill and non-critically ill AIDS patients. Antimicrob Agents Chemother. 1995;39:28-33.

29. Bushby SR, Hitchings GH. Trimethoprim, a sulphonamide potentiator. Br J Pharmacol Chemother. 1968;33:72-90.

30. Bergan T, Brodwall EK. The pharmacokinetic profile of co-trimoxazole. Scand J Infect Dis Suppl. 1976;8:42-9.

31. Cirioni O, Giacometti A, Scalise G. In-vitro activity of atovaquone, sulphamethoxazole and dapsone alone and combined with inhibitors of dihydrofolate reductase and macrolides against Pneumocystis carinii. J Antimicrob Chemother. 1997;39:45-51.

32. Craig WA, Kunin CM. Distribution of trimethoprim-sulfamethoxazole in tissues of rhesus monkeys. J Infect Dis. $1973 ; 128: 575-9$.

33. Dubar V, Lopez I, Gosset P, Aerts C, Voisin C, Wallaert B. The penetration of co-trimoxazole into alveolar macrophages and its effect on inflammatory and immunoregulatory functions. J Antimicrob Chemother. 1990;26:791-802.

34. Nuesch R, Bellini C, Zimmerli W. Pneumocystis carinii pneumonia in human immunodeficiency virus (HIV)-positive and HIV-negative immunocompromised patients. Clin Infect Dis. 1999;29:1519-23.

35. Sepkowitz KA. Opportunistic infections in patients with and patients without acquired immunodeficiency syndrome. Clin Infect Dis. 2002;34:1098-107. 